\title{
OPERATOR METHOD IN SOLVING NON-LINEAR EQUATIONS OF THE HARTREE-FOCK TYPE
}

\author{
Le Anh Thu ${ }^{* 1}$ and L I Komarov ${ }^{2}$ \\ 1 Institute of Physics, P.O. Box 429 Bo Ho, Hanoi 10000, Vietnam \\ ${ }^{2}$ Department of Physics, Belarussian State University, 4 Fr. Skariny av., Minsk 220050, Republic of Belarus
}

(October 26, 2018)

\begin{abstract}
The operator method is used to construct the solutions of the problem of the polaron in the strong coupling limit and of the helium atom on the basis of the Hartree-Fock equation. $E_{0}=$ $-0.1085128052 \alpha^{2}$ is obtained for the polaron ground-state energy. Energies for $2 s$ - and $3 s$-states are also calculated. The other excited states are briefly discussed.
\end{abstract}

\section{INTRODUCTION}

The operator method (OM) of the approximate solution of the Schrödinger equation was suggested in the works of Feranchuk and Komarov [1-3]. In agreement with the results obtained in these and subsequent works (see, for example, refs. [4-12]), the solution in the zeroth approximation of the OM gives quite a simple and universal algorithm for obtaining an approximation, which is uniformly applicable in a wide range of variation of parameters of the Hamiltonian. Another advantage of the OM is connected with the possibility of regularly calculating the correction to the zeroth approximation. Here, in any order the needed calculations are reduced to a simple algebraic procedure of expressing the product of excitation creation and annihilation operators in the normal form, and this essentially simplified the use of computers.

As shown by the results of many works the OM is very effective for solving various physical problems. However, the question of the application of this method in solving non-linear equations, which are often met in atomic and solid state physics, remains unclear at present.

In this work we investigate in two examples the possibility of using the OM for solving non-linear equations such as that of Hartree-Fock:

(i) in an example of the problem of a polaron in the regime of strong electron-phonon coupling (section II);

(ii) in an example of the problem of a helium atom (section III).

It should be noted that such calculations have been carried out on the basis of various methods. But here, we want to obtain the exact solution by a numerical method. Comparison of approximate solutions with the exact numerical solution enables us to estimate not only the accuracy of the approximate solutions, but also the usefulness of their further study.

\section{THE OPERATOR METHOD IN THE POLARON PROBLEM}

The problem of a polaron in the strong coupling regime has become of interest since Emin [13] proposed the mechanism of bipolaron Bose-Einstein condensation to explain high- $T_{c}$ superconductivity, and on the other hand, the bipolaron is known to exist in this strong coupling regime [14. The Hamiltonian of a non-relativistic particle (electron) interacting with the quantized scalar field of lattice oscillations in an ionic crystal has the form (see, for example, refs. $[6,7,15,16]$ and reviews $[17,18])$ :

$$
\hat{H}=-\frac{\triangle}{2 m}+\sum_{\mathbf{k}} \omega_{\mathbf{k}} c_{\mathbf{k}}^{+} c_{\mathbf{k}}+\frac{g}{\sqrt{\Omega}} \sum_{\mathbf{k}}\left(A_{\mathbf{k}} e^{i \mathbf{k r}} c_{\mathbf{k}}+A_{\mathbf{k}}^{*} e^{-i \mathbf{k r}} c_{\mathbf{k}}^{+}\right)
$$

Here, $c_{\mathbf{k}}^{+}$and $c_{\mathbf{k}}$ are respectively the operators of creation and annihilation of phonons with frequency $\omega_{\mathbf{k}}$ and momentum $\mathbf{k}, A_{k}$ are the Fourier components of the source density and $\Omega$ is the volume of the system. In the theory of

\footnotetext{
*Present address: Max-Planck Institute for Physics of Complex Systems, Nöthnitzer Str. 38, D-01187 Dresden, Germany. E-mail: lathu@idefix.mpipks-dresden.mpg.de
} 
the so-called optical polaron, the frequency of phonons is assumed to be independent of their wavevector. Usually, it is assumed that

$$
\omega_{\mathbf{k}}=\omega_{0}, \quad g A_{\mathbf{k}}=-\frac{i \omega_{0}}{k} \frac{(4 \pi \alpha)^{1 / 2}}{\left(2 m \omega_{0}\right)^{1 / 4}},
$$

where $\alpha$ is a dimensionless electron-phonon coupling constant.

For the application of the $\mathrm{OM}$ it is conducive to cross to the four-dimensional oscillator representation using the transformation [19]

$$
\left\{\begin{array}{l}
x_{\lambda}=\xi_{s}^{*}\left(\sigma_{\lambda}\right)_{s t} \xi_{t} \\
\chi=\arg \left(\xi_{1}\right)
\end{array}\right.
$$

where $\sigma_{\lambda}(\lambda=1,2,3)$ are Pauli matrixes, $\xi_{s}(s=1,2)$ are regarded as spinor components. The physical meaning of "extra" variable $\chi$ has been treated in many works (see, for example, refs. [20,21] and references therein).

Let us define operators

$$
\begin{array}{ll}
a_{s}(\omega)=\sqrt{\frac{\omega}{2}}\left(\xi_{s}+\frac{1}{\omega} \frac{\partial}{\partial \xi_{s}^{*}}\right), & b_{s}(\omega)=\sqrt{\frac{\omega}{2}}\left(\xi_{s}^{*}+\frac{1}{\omega} \frac{\partial}{\partial \xi_{s}}\right), \\
a_{s}^{+}(\omega)=\sqrt{\frac{\omega}{2}}\left(\xi_{s}^{*}-\frac{1}{\omega} \frac{\partial}{\partial \xi_{s}}\right), & b_{s}^{+}(\omega)=\sqrt{\frac{\omega}{2}}\left(\xi_{s}-\frac{1}{\omega} \frac{\partial}{\partial \xi_{s}^{*}}\right) .
\end{array}
$$

Here, the positive parameter $\omega$ will be defined later. Operators (2) satisfy the commutation relations

$$
\left[a_{s}(\omega), a_{t}^{+}(\omega)\right]=\delta_{s t}, \quad\left[b_{s}(\omega), b_{t}^{+}(\omega)\right]=\delta_{s t}
$$

(we have written only non-zero commutators). The possibility of using the algebraic method is conditioned by the fact that, all "physical" operators can be expressed through the following 15 operators

$$
\begin{gathered}
M=a_{s} b_{s}, \quad M^{+}=a_{s}^{+} b_{s}^{+}, \quad N=a_{s}^{+} a_{s}+b_{s}^{+} b_{s}, \\
m_{\lambda}=\left(\sigma_{\lambda}\right)_{s t} a_{t} b_{s}, \quad m_{\lambda}^{+}=\left(\sigma_{\lambda}\right)_{s t} a_{s}^{+} b_{t}^{+}, \quad n_{\lambda}^{a}=\left(\sigma_{\lambda}\right)_{s t} a_{s}^{+} a_{t}, \quad n_{\lambda}^{b}=\left(\sigma_{\lambda}\right)_{s t} b_{t}^{+} b_{s},
\end{gathered}
$$

which form a closed algebra $s o(4,2)$ (see ref. 19]). Here and henceforth, we omit for brevity the parameter $\omega$ in expressions of the operators. For further use we rewrite some operators into this representation as follows

$$
\begin{gathered}
x_{\lambda}=\frac{1}{2 \omega}\left(m_{\lambda}^{+}+m_{\lambda}+n_{\lambda}^{a}+n_{\lambda}^{b}\right), \quad r=\xi_{s}^{*} \xi_{s}=\frac{1}{2 \omega}\left(M^{+}+M+N+2\right), \\
r \triangle=-\frac{\partial^{2}}{\partial \xi_{s}^{*} \partial \xi_{s}}=\frac{\omega}{2}\left(2+N-M-M^{+}\right),
\end{gathered}
$$

where the operators $N, M, M^{+}$form the subalgebra $s o(2,1)$ and satisfy the following relations

$$
\left[M, M^{+}\right]=2+N, \quad[M, 2+N]=2 M, \quad\left[2+N, M^{+}\right]=2 M^{+} .
$$

In the four-dimensional oscillator representation ( $\xi$-space) the equation for eigenvalue problem $\hat{H}|\Psi\rangle=E|\Psi\rangle$ has the form [19]

$$
\hat{L}|\Psi\rangle \equiv r(\hat{H}-E)|\Psi\rangle=0
$$

which, in terms of the operators (3), rather simplifies the algebraic calculation of the matrix elements. It is easy to see that this representation is especially useful for the bound polaron problem and leads to the equation without singular Coulomb term. Further, we shall limit ourselves to case of the strong coupling regime, i.e. when $\alpha \gg 1$. It is a well known fact that, in this limit, one can neglect the quantum fluctuations of the phonon field (see [17] and a recent discussion in [22]). After introducing the canonical transformations

$$
c_{\mathbf{k}}=b_{\mathbf{k}}+u_{\mathbf{k}}, \quad c_{\mathbf{k}}^{+}=b_{\mathbf{k}}^{+}+u_{\mathbf{k}}^{*}
$$


and neglecting the quantum components, we then have

$$
\hat{L}=-\frac{1}{2} r \Delta+2^{3 / 4}\left(\frac{\pi \alpha}{\Omega}\right)^{1 / 2} \sum_{\mathbf{k}} \frac{1}{k}\left(r e^{i \mathbf{k r}} u_{\mathbf{k}}+r e^{-i \mathbf{k r}} u_{\mathbf{k}}^{*}\right)+r\left(\sum_{\mathbf{k}} u_{\mathbf{k}}^{*} u_{\mathbf{k}}-E\right)
$$

(here, all measurements are in the system of units whereby $m=\hbar=\omega_{0}=1$ ). The classical components of the field can be defined from the condition

$$
\frac{\partial E}{\partial u_{\mathbf{k}}}=\frac{\partial E}{\partial u_{\mathbf{k}}^{*}}=0
$$

From equations (5), (7) and (8) we obtain

$$
u_{\mathbf{k}}=-2^{3 / 4}\left(\frac{\pi \alpha}{\Omega}\right)^{\frac{1}{2}} \frac{1}{\langle r\rangle k}\left\langle r e^{-i \mathbf{k r}}\right\rangle .
$$

Here $\langle\ldots\rangle$ represents the average $\langle\Psi|\ldots| \Psi\rangle$, where $|\Psi\rangle$ is the polaron eigenfunction. Substituting (9) into (7) and than integrating over $\mathbf{k}$ we have

$$
\hat{L}=-\frac{1}{2} r \triangle-2^{1 / 2} \alpha \frac{1}{\langle r\rangle} \int d^{4} \eta \frac{r r^{\prime}|\Psi(\eta)|^{2}}{\left|\mathbf{r}-\mathbf{r}^{\prime}\right|}+r\left(\frac{\alpha}{2^{1 / 2}\langle r\rangle^{2}} \int d^{4} \eta \int d^{4} \zeta \frac{r^{\prime} r^{\prime \prime}}{\left|\mathbf{r}^{\prime}-\mathbf{r}^{\prime \prime}\right|}|\Psi(\eta)|^{2}|\Psi(\zeta)|^{2}-E\right),
$$

where

$$
x_{\lambda}^{\prime}=\eta_{s}^{*}\left(\sigma_{\lambda}\right)_{s t} \eta_{t}, \quad x_{\lambda}^{\prime \prime}=\zeta_{s}^{*}\left(\sigma_{\lambda}\right)_{s t} \zeta_{t} .
$$

The emergence of the terms $\langle r\rangle$ and $\langle r\rangle^{2}$ is conditioned by the changes in the condition of normalization of wavefunctions in the $\xi$-space. Further, we shall consider only $s$-states of the polaron. The general case will be treated at the end of this paper. Given the fact that the eigenfunctions of $s$-states spherically symmetric, i.e. they depend only on $r$, we can write $\Psi(\xi) \equiv \Psi(r)$. Averaging (10) over the angles and using the formula

$$
\frac{1}{2} \int_{-1}^{1} d x \frac{1}{\sqrt{r^{2}+r^{\prime 2}-2 x r r^{\prime}}}=\frac{1}{r r^{\prime}}\left[r^{\prime} \theta\left(r-r^{\prime}\right)+r \theta\left(r^{\prime}-r\right)\right]
$$

where the Heaviside function $\theta(x)$ vanishes for $x<0$, is $1 / 2$ for $x=0$ and unity for $x>0$, we have

$$
\begin{gathered}
\left(-\frac{1}{2} r \triangle-\sqrt{2} \alpha \frac{1}{\langle r\rangle} \int d^{4} \eta|\Psi(\eta)|^{2}\left[r^{\prime} \theta\left(r-r^{\prime}\right)+r \theta\left(r^{\prime}-r\right)\right]+\right. \\
\left.+\frac{\alpha}{\sqrt{2}\langle r\rangle^{2}} r \int d^{4} \eta \int d^{4} \zeta|\Psi(\eta)|^{2}|\Psi(\zeta)|^{2}\left[r^{\prime} \theta\left(r^{\prime \prime}-r^{\prime}\right)+r^{\prime \prime} \theta\left(r^{\prime}-r^{\prime \prime}\right)\right]-r E\right)|\Psi(\xi)\rangle=0 .
\end{gathered}
$$

It is clear that equation (11) is an integro-differential equation of the Hartree-Fock type. This equation can be simplified using new units of energy and length, such units are equal to the old ones multiplied respectively by $\alpha^{2}$ and $1 / \alpha$. In this units the parameter $\alpha$ disappears in the last equation. Therefore, we can further put $\alpha=1$.

Let assume that the polaron eigenfunction has the form

$$
|\Psi\rangle=\sum_{n} C_{n}|n\rangle
$$

where

$$
|n\rangle=\frac{1}{\sqrt{n !(n+1) !}}\left(M^{+}\right)^{n}|0\rangle .
$$

It is clear that we need to calculate the matrix elements of the operators constituting $\hat{L}$, such as

$$
\left\langle m_{1}, m_{2}\left|\left[r_{2} \theta\left(r_{1}-r_{2}\right)+r_{1} \theta\left(r_{2}-r_{1}\right)\right]\right| n_{1}, n_{2}\right\rangle \equiv W_{m_{1} m_{2} ; n_{1} n_{2}} .
$$


To this effect we use the integral representation

$$
r_{2} \theta\left(r_{1}-r_{2}\right)+r_{1} \theta\left(r_{2}-r_{1}\right)=\left(\frac{1}{2 \pi}\right)^{2} \int_{-\infty}^{+\infty} d q_{1} \int_{-\infty}^{+\infty} d q_{2} e^{i q_{1} r_{1}+i q_{2} r_{2}} \varphi\left(q_{1}, q_{2}\right)
$$

where

$$
\varphi\left(q_{1}, q_{2}\right)=\int_{0}^{\infty} d r_{1} \int_{0}^{\infty} d r_{2}\left[r_{2} \theta\left(r_{1}-r_{2}\right)+r_{1} \theta\left(r_{2}-r_{1}\right)\right] e^{-i q_{1} r_{1}-i q_{2} r_{2}} .
$$

The operator

$$
\exp (i q r)=\exp \left(\frac{i q}{2 \omega}\left(2+N+M+M^{+}\right)\right)
$$

can be expressed in the normal form as follows (see, for example, [19], 23])

$$
\exp (i q r)=\frac{1}{(1-\mu)^{2}} \exp \left(\frac{1}{1-\mu} M^{+}\right) \exp (-N \ln (1-\mu)) \exp \left(\frac{1}{1-\mu} M\right)
$$

where, $\mu=\frac{i q}{2 \omega}$. As a result, the calculation of matrix elements of the operator $\exp (i q r)$ using the algebra (4) does not pose much difficulties. Here, we give only the result obtained

$$
\langle m|\exp (i q r)| n\rangle=\sqrt{\frac{n+1}{m+1}} \sum_{s=0}^{n} C_{n}^{s} C_{n}^{s+1} \frac{(\mu)^{m+n-2 s}}{(1-\mu)^{m+n+2}},
$$

where

$$
C_{q}^{p}=\frac{q !}{p !(q-p) !}
$$

Subtituting (15) into (13) and (14), after integration over the variables $q_{1}, q_{2}, r_{1}, r_{2}$ we finally have the formula

$$
\begin{gathered}
W_{m_{1} m_{2} ; n_{1} n_{2}}=\frac{1}{16 \omega}(-1)^{m_{1}+m_{2}+n_{1}+n_{2}} \sqrt{\frac{\left(n_{1}+1\right)\left(n_{2}+1\right)}{\left(m_{1}+1\right)\left(m_{2}+1\right)}} \sum_{s_{1}=0}^{n_{1}} C_{n_{1}}^{s_{1}} C_{m_{1}+1}^{s_{1}+1} \\
\times \sum_{t_{1}=0}^{m_{1}+n_{1}-2 s_{1}}(-1)^{t_{1}} C_{m_{1}+n_{1}-2 s_{1}}^{t_{1}} \sum_{s_{2}=0}^{n_{2}} C_{n_{2}}^{s_{2}} C_{m_{2}+1}^{s_{2}+1} \sum_{t_{2}=0}^{m_{2}+n_{2}-2 s_{2}}(-1)^{t_{2}} C_{m_{2}+n_{2}-2 s_{2}}^{t_{2}} \\
\times\left(2 s_{1}+t_{1}+2 s_{2}+t_{2}+3\right)\left(2 s_{1}+t_{1}+2 s_{2}+t_{2}+4\right) C_{2 s_{1}+t_{1}+2 s_{2}+t_{2}+2}^{2 s_{1}+t_{1}+1} \\
\times\left\{\sum_{u=0}^{2 s_{1}+t_{1}+1} \frac{(-1)^{u} C_{2 s_{1}+t_{1}+1}^{u}}{\left(2 s_{2}+t_{2}+3+u\right) 2^{2 s_{2}+t_{2}+u}}+\sum_{u=0}^{2 s_{2}+t_{2}+1} \frac{(-1)^{u} C_{2 s_{2}+t_{2}+1}^{u}}{\left(2 s_{1}+t_{1}+3+u\right) 2^{2 s_{1}+t_{1}+u}}\right\} .
\end{gathered}
$$

Other matrix elements are calculated in the same way.

Let us now consider the zeroth approximation of the OM. The free parameter $\omega$ is chosen in such a way that the condition

$$
\frac{\partial E^{(0)}}{\partial \omega}=0
$$

is satisfied [3]. Whence

$$
\omega_{n}^{(0)}=\frac{W_{n n n n}}{2 \sqrt{2}(n+1)^{2}}, \quad E_{n}^{(0)}=-\frac{W_{n n n n}^{2}}{16(n+1)^{4}} .
$$


For some first $s$-states these equations give the following results

$$
E_{0}^{(0)}=-0.097656 \alpha^{2}, \quad E_{1}^{(0)}=-0.0226 \alpha^{2}, \quad E_{2}^{(0)}=-0.0099 \alpha^{2},
$$

which are different from the exact ones approximately by $10 \%$.

Equation (11) is a special case of the following generalized equation (see refs. [9,12])

$$
\left(\hat{A}-E_{n} \hat{B}\right)\left|\Psi_{n}\right\rangle=0 .
$$

Therefore, we describe here a general iteration scheme for the last equation. We shall find the eigenfunction in the form

$$
\left|\Psi_{n}\right\rangle=|n\rangle+\sum_{k \neq n} c_{k n}|k\rangle
$$

The substitution of this expression into equation (17) gives the system of equations ( $s$ is a number of iterations)

$$
\begin{gathered}
E_{n}^{(s)}=\left(B_{n n}+\sum_{k \neq n} c_{k n}^{(s)} B_{n k}\right)^{-1}\left(A_{n n}+\sum_{k \neq n} c_{k n}^{(s)} A_{n k}\right), \\
c_{i n}^{(s)}=-\left(A_{i i}-E_{n}^{(s-1)} B_{i i}\right)^{-1} \sum_{k \neq n, i}\left(A_{i k}-E_{n}^{(s-1)} B_{i k}\right) c_{k n}^{(s-1)},
\end{gathered}
$$

where the eigenvalue $E_{n}$ and the coefficients of the eigenfunctions $c_{k n}$ are defined by the relations

$$
E_{n}=\lim _{s \rightarrow \infty} E_{n}^{(s)}, \quad c_{k n}=\lim _{s \rightarrow \infty} c_{k n}^{(s)} .
$$

Equations (18)-(21) completely define quite a simple algorithm for solving equation (17). Apparently, the nondiagonal matrix elements of the Hamiltonian are "included" in each step of the subsequent iteration. It should be noted that there exist some other iteration schemes, for example, an interesting variational-iterative one, proposed by Burrows and Core (for more details, see [24]). Here we use the above scheme (18)-(21) because of its simplicity and transparency. Moreover, this and some other similar schemes proved to be well convergent in many applications of the OM (see $[8,9,12]$ and references therein).

The results of our calculation show good convergence of the OM in quite a wide range of variation of the parameter $\omega$. For $s=18$ we obtain the following value for the polaron ground-state energy

$$
E_{0}=-0.1085128052 \alpha^{2} \text {. }
$$

This result is in good agreement with the numerical solution, obtained by Miyake [25] and confirmed by Adamowski et al [26]. It should be noted that good numerical results have been obtained recently by a variational method, based on the coherent state representations [27]. We would like to point out that the OM enables one also to find with high accuracy the energy and eigenfunction of the excited states, which usually are obtained with much more difficulty using other methods [28]- 31]. We present here the value of the energy for $2 s$ - and $3 s$-states (for $s=18$ )

$$
E_{1}=-0.02053101 \alpha^{2}, \quad E_{2}=-0.0083506 \alpha^{2} .
$$

Our results are displayed in table 1 together with the known results for a polaron in the strong coupling limit.

Table 1. Polaron energy (in $\alpha^{2} \hbar \omega_{0}$ units) for $s$-states in the strong coupling limit.

\begin{tabular}{llll}
\hline \hline \multicolumn{1}{c}{ Authors } & \multicolumn{1}{c}{$1 s$} & $2 s$ & $3 s$ \\
\hline Pekar (by Miyake [25]) & -0.108504 & & \\
Miyake [25] & -0.108513 & & \\
Adamowski et al [26] & -0.1085128 & & \\
Feranchuk et al [2] & -0.1078 & & \\
Smondyrev [32] & -0.109206 & & \\
Efimov et al [15] & -0.10843 & & \\
Ganbold et al [16] & -0.107766 & & \\
Chen et al [27] & -0.10851 & & \\
Hagen et al [30] & & -0.02048 & -0.00804 \\
Balabaev et al [31] & & -0.0206 & -0.00832 \\
Our results & -0.1085128052 & -0.02053101 & -0.0083506 \\
\hline \hline
\end{tabular}




\section{THE OPERATOR METHOD IN SOLVING THE HARTREE-FOCK EQUATION FOR HELIUM}

Let us now consider the problem of helium. For the ground state, we have the following Hartree-Fock equation (see, for example, ref. [33])

$$
\left[-\frac{1}{2} \triangle_{1}-E_{1}-\frac{Z}{r_{1}}+\int \frac{\Psi^{2}\left(\mathbf{r}^{\prime}\right)}{\left|\mathbf{r}_{1}-\mathbf{r}^{\prime}\right|} d \mathbf{r}^{\prime}\right] \Psi\left(r_{1}\right)=0
$$

(for the second electron, the corresponding equation is written in analogy with the first).

The energy of the atom is

$$
E=2 E_{1}-\iint \frac{\Psi^{2}\left(\mathbf{r}_{1}\right) \Psi^{2}\left(\mathbf{r}_{2}\right)}{\left|\mathbf{r}_{1}-\mathbf{r}_{2}\right|} d \mathbf{r}_{1} d \mathbf{r}_{2}
$$

The substitution of the last expression into equation (22) gives

$$
\left[-\frac{1}{2} \triangle-\frac{Z}{r}+\int \frac{\Psi^{2}\left(\mathbf{r}^{\prime}\right)}{\left|\mathbf{r}-\mathbf{r}^{\prime}\right|} d \mathbf{r}^{\prime}-\frac{1}{2} \iint \frac{\Psi^{2}\left(\mathbf{r}_{1}\right) \Psi^{2}\left(\mathbf{r}_{2}\right)}{\left|\mathbf{r}_{1}-\mathbf{r}_{2}\right|} d \mathbf{r}_{1} d \mathbf{r}_{2}-\frac{E}{2}\right] \Psi(r)=0 .
$$

In $\xi$-space the equation (23) has the form

$$
\begin{gathered}
\left\{-\frac{1}{2} r \Delta-Z+\frac{1}{\langle r\rangle} \int d^{4} \eta \Psi^{2}(\eta)\left[r^{\prime} \theta\left(r-r^{\prime}\right)+r \theta\left(r^{\prime}-r\right)\right]-\right. \\
\left.-\frac{r}{2}\left(\frac{1}{\langle r\rangle^{2}} \int d^{4} \eta \int d^{4} \zeta \Psi^{2}(\eta) \Psi^{2}(\zeta)\left[r^{\prime} \theta\left(r^{\prime \prime}-r^{\prime}\right)+r^{\prime \prime} \theta\left(r^{\prime}-r^{\prime \prime}\right)\right]+E\right)\right\} \Psi(\xi)=0 .
\end{gathered}
$$

The last equation has the same form as equation (11), for which we have already calculated the matrix elements. The key difference is that now we have a negative "coupling constant" $\alpha$. In order to have the needed solutions, we need only to change the formulae of the iteration scheme. Consequently, for $s=18$ we have (in atomic units)

$$
E^{H F}=-2.861679995
$$

which should be compared with the known result [33] $E^{H F}=-2.863$ and with a recent calculation [34] $E^{H F}=$ -2.861680 . It follows that the OM gives the solutions with very high accuracy independently of the repulsive or attractive nature of the interaction.

\section{CONCLUSIONS}

In this paper we have applied an algebraic approach, namely, the OM to solve the non-linear equations, which appear in the problem of polaron in the strong coupling limit and the helium atom in the Hartree-Fock approximation. The resuls are as good as the best ones in the literature. Furthermore, we would like to point out that the present approach should be successfully applied to the other polaron problems, such as bound polarons and polarons in external fields in 3D-space as well as in the other dimensions.

Finally, we note the following two circumstances:

(i) As shown in ref. [35], the equation, which describes the motion of an individual particle in a system of large number of gravitational bosons in fact coincides with equation (11). Therefore, the solutions obtained here for the polaron are simultaneously solutions for the above-mentioned problem.

(ii) We need not here only consider $s$-states. To this effect it is conducive to use in equation (10) the following integral representation

$$
\frac{1}{\left|\mathbf{r}-\mathbf{r}^{\prime}\right|}=\frac{1}{2 \pi} \int_{0}^{2 \pi} d \chi \frac{1}{\left(\xi_{s}^{*}-\eta_{s}^{*}\right)\left(\xi_{s}-\eta_{s}\right)} .
$$

Further calculation of matrix elements using this formula and the algebra so $(4,2)$ of operators $(3)$ does not pose much difficulty and is affected in analogy with the calculations carried out above. 
[1] Feranchuk I D and Komarov L I 1982 Phys. Lett. A 88212

[2] Feranchuk I D and Komarov L I 1982 J. Phys. C 151965

[3] Feranchuk I D and Komarov L I 1984 J. Phys. A 173111

[4] Gerry C C and Silverman S 1983 Phys. Lett. A 95481

[5] Witchel W 1983 Phys. Lett. A 97315

[6] Feranchuk I D, Fisher S I and Komarov L I 1984 J. Phys. C 174309

[7] Feranchuk I D, Fisher S I and Komarov L I 1985 J. Phys. C 185083

[8] Fernandez F M and Castro E A 1985 Phys. Lett. A 111104

[9] Fernandez F M, Castro E A and Meson A M 1986 Mol. Phys. 58365

[10] An C Z, Feranchuk I D and Komarov L I 1987 Phys. Lett. A 125123

[11] Feranchuk I D, Kuz'min V S and Ulyanenkov A P 1991 Chem. Phys. 15761

[12] Le Anh Thu, Hoang Quoc Khanh and Komarov L I 1994 Int. Workshop "Quantum Systems: New Trends and Methods", Minsk, May 23-29, unpublished

[13] Emin D 1989 Phys. Rev. Lett. 62 1544; 1992 Phys. Rev. B 455525

[14] Smondyrev M A, Devreese J T and Peeters F M 1995 Phys. Rev. B 5115008

[15] Efimov G V and Ganbold G 1991 Phys. Stat. Sol. B 168165

[16] Ganbold G and Efimov G V 1994 Phys. Rev. B 503733

[17] Mitra T K, Chatterjee A and Mukhopadhyay S 1987 Phys. Rep. 15391

[18] Lakhno V D and Chuev G N 1995 Sov. Phys. Uspekhi 38273

[19] Komarov L I and Romanova T S 1982 Izv. Akad. Nauk BSSR (ser. Fiz.-Mat. Nauk) 298 (in Russian); 1985 J. Phys. B: At. Mol. Phys. 18859

[20] Le Van Hoang and Viloria T 1992 Phys. Lett. A 17123

[21] Le Van Hoang, Ly Xuan Hai, Komarov L I and Romanova T S 1992 J. Phys. A: Math. Gen. 256461

[22] Lieb E H and Thomas L E 1997 Commun. Math. Phys. 183 511; ibid. 188499

[23] Wilcox R M 1967 J. Math. Phys. 8962

[24] Burrows B L and Core P W 1984 J. Phys. A: Math. Gen. 17559

[25] Miyake S J 1975 J. Phys. Soc. Jap. 38181

[26] Adamowski J, Gerlach B and Leschke H 1980 Phys. Lett. A 79249

[27] Chen Q, Fang M, Zhang Q, Wang K and Wan S 1996 J. Phys.:Condens. Matter 87139

[28] Gabdoulline R R 1993 Dok. Ak. Nauk RAN 33323 (in Russian)

[29] Chuev G N and Lakhno V D 1993 Phys. Rev. B 4812655

[30] Hagen D E, Van Zandt L L and Prohofsky E W 1970 Phys. Rev. B 2553

[31] Balabaev N K and Lakhno V D 1980 Sov. Theor. Math. Phys. 45936

[32] Smondyrev M A 1991 Physica A 171191

[33] Bethe H A and Salpeter E E 1977 Quantum Mechanics of One- and Two Electron Atoms (New York: Plenum)

[34] Styszynski J and Karwowski J 1988 J. Phys. B: At. Mol. Opt. Phys. 21 2389; Fischer C F 1987 Comput. Phys. Commun. 43335

[35] Komarov L I 1984 Izv. Akad. Nauk BSSR (ser. Fiz.-Mat. Nauk) 390 (in Russian) 